\title{
Performance Analysis of Directional Cooperative Communication System
}

\author{
R.-L. Tan \\ 1) The University of Armed Police Engineering, Xi’an, Shaanxi, China (madamtan@126.com) \\ ${ }^{2)}$ Research Center of Scientific Research Departments for Equipment development and Application, The University of Air Force \\ Engineering, Xi’an, Shanxi, China (madamtan@126.com)
}

\begin{abstract}
For specific military use of the environment, this paper study the performance change of the system used directional antennas and exploratory study the power optimization based on the symbol error rate(SER) under amplify-and-forward protocol (AF). Firstly, cooperative communication system model based on the directional antenna was established, and then the closed-form expression of the model was derived theoretically. Secondly, it analyzed the optimal power allocation (OPA) problem in order to minimize system SER. Finally, it analyzed and compared the performance of the SER combining with specific numerical and simulation in detail. The results showed that in the condition of using directional transmission and directional reception, system had smaller SER. Directional gain, channel quality and power distribution of each node had enormous impact on the whole system performance. OPA had better performance than equal power allocation (EPA).
\end{abstract}

Keywords - Cooperative communication, Directional antenna, Amplify-and-forward protocol, Symbol error rate

\section{定向协作通信系统性能分析}

\author{
檀荵莲 \\ 1) 西安武警工程大学，西安，陕西，中国 \\ 2) 空军工程大学科研部装备发展与运用研究中心，西安，陕西，中国
}

摘＼cjkstart要＼cjkstart针对特定的军事使用环境，研究引入定向天线后系统的性能变化，并以放大转发协议（Amplify-and-Forward Protocol, AF）下的误符号率（Symbol Error Rate, SER）为研究对象探讨功率优化问题。首先建立了引入定向天线的协作通信系统模型，从理 论上推导出该模型下的 SER 闭式表达式, 并以最小化系统 SER 为目标分析最优功率分配问题, 最后结合具体的仿真数值对所建立模 型的误符号性能进行详细分析比较。仿真结果表明, 协作通信系统收发端在采用定向发送定向接收的条件下, 误符号率更小, 各节点 定向增益、信道质量和功率分配方式对系统整体性能影响巨大，最优功率分配比等功率分配性能更优。

关键词 协作通信，定向天线，放大转发，误符号率

1. 引言

协作通信作为近几年的研究热点，取得了许多阶段 性的成果[1-6]。定向天线能够把能量集中发射到同一个 方向上, 不但可以降低其他方向的干扰, 同时能够增加 空间复用, 在相同的发送功率下传输范围更广, 因此在 军事领域得到广泛应用[7-9]。例如美军在 F-22 战斗机上 安装了 6 组定向天线, 覆盖整个空域从而实现了射频隐 身的目的。虽然关于协作通信和定向天线的研究很多, 但是将二者结合研究鲜有文献涉及[10], 在军事通信领
域特定的应用背景下, 将二者结合研究具有重大的理论 意义与应用价值。

本文将定向天线引入协作通信系统进行探索性研 究。通过数学建模与理论推导, 得到引入定向天线后系 统的 SER 闭式表达形式并确定其上界, 进一步分析功率 分配对系统 SER 的影响。发现在特定的环境中, 引入定 向天线的通信系统性能更优越，同时，系统性能受各节 点定向增益和信道质量影响很大, 根据不同的信道环境 合理进行功率分配可以大大提高系统性能。 


\section{2. 基于定向天线的协作通信系统模型}

定向天线接收信号可表达如下[10]:

$$
P_{f}=\frac{P_{t} G_{t} G_{r}}{K r^{v}}
$$

其中, $P_{t}$ 表示发射功率, $G_{t}$ 表示发射端的定向天线 增益, $G_{r}$ 表示接收端定向天线增益, $K$ 为关于大气吸收, 欧姆损耗等的常数, $v$ 为路径衰落系数。

鉴于定向天线波束成型后信号能量只能覆盖一定的 空间角度, 假设从源节点到中继节点及目的节点的角度 $\theta$ (如图 1 所示) 大于波束成型角度, 那么源节点就需 要用 2 个时隙分别给中继和目的节点发送消息，与传统 的协作通信相比将要多消耗 1 个通信时隙 $[10]$ 。若以 $h_{i, j}$ 表示各节点的信道系数, 则其服从参数为 $1 / \sigma_{i, j}^{2}$ 的指数分 布。此时, 整个通信过程分三个时隙两个阶段完成, 如 图 1 所示:

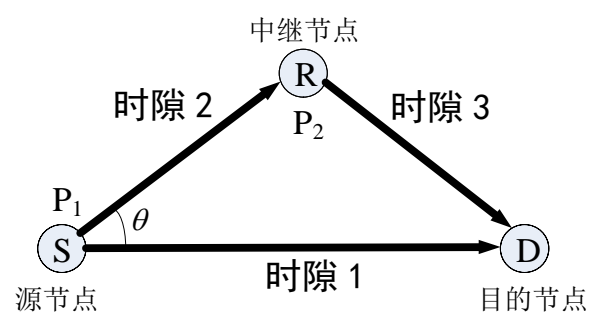

图 1 定向协作通信系统模型

阶段 1: 源节点分别向目的节点和中继节点发送广 播信息。设目的节点与中继节点接收到的信息分别为 $y_{s, d}, y_{s, r}$, 用 $G_{s}, G_{r}, G_{d}$ 分别表示源节点, 中继节点 和目的节点的定向天线增益，则:

$$
\begin{aligned}
& y_{s, d}=\sqrt{P_{1} G_{s} G_{d}} h_{s, d} x+\eta_{s, d} \\
& y_{s, r}=\sqrt{P_{1} G_{s} G_{r}} h_{s, r} x+\eta_{s, r}
\end{aligned}
$$

其中, $x$ 为发送的信号符号, $P_{1}$ 为源的发射功率, $\eta_{s, d}, \eta_{s, r}$ 是均值为零, 方差为 $N_{o}$ 的加性高斯白噪声; $h_{s, d}$, $h_{s, r}$ 分别为源到目的节点和源到中继节点的信道系数, 分 别为服从均值为零, 方差为 $\sigma_{s, d}{ }^{2}$ 和 $\sigma_{s, r}{ }^{2}$ 的复高斯随机变 量, 本文设定方差均不为零。

阶段 2: AF 协议下中继将接收到的信号放大转发给 目的节点。设中继端的发射功率为 $P_{2}$, 则在放大因子 $\beta=\sqrt{P_{2} G_{r} G_{d}} / \sqrt{P_{1} G_{s} G_{r}\left|h_{s, r}\right|^{2}+N_{0}}$ 的情况下, 目的端接收 到中继的信号可表示为:

$$
y_{r, d}=\beta h_{r, d} y_{s, r}+\eta_{r, d}
$$

将式 (3) 和 $\beta$ 的具体数值代入式 (4) 整理得到:

$$
y_{r, d}=\frac{\sqrt{P_{1} P_{2} G_{s} G_{r}{ }^{2} G_{d}}}{\sqrt{P_{1} G_{s} G_{r}\left|h_{s, r}\right|^{2}+N_{0}}} h_{r, d} h_{s, r} x+\tilde{\eta}_{r, d}
$$

其中, $\tilde{\eta}_{r, d}=\frac{\sqrt{P_{2} G_{r} G_{d}}}{\sqrt{P_{1} G_{s} G_{r}\left|h_{s, r}\right|^{2}+N_{0}}} h_{r, d} \eta_{s, r}+\eta_{r, d}$ 。设 $\eta_{s, d}, \eta_{s, r}$ 独 立, 则等效噪声 $\tilde{\eta}_{r, d}$ 是均值为零的复高斯随机变量, 方 差为:

$$
\tilde{N}_{0}=\left(\frac{P_{2} G_{r} G_{d}\left|h_{r, d}\right|^{2}}{P_{1} G_{s} G_{r}\left|h_{s, r}\right|^{2}+N_{0}}+1\right) N_{0}
$$

目的端通常采用最大比合并（Maximum Ratio Combining, MRC) 方法, 则第一路是 $y_{s, d}$, 设其信噪比 为 $\gamma_{1}$, 第二路是 $y_{r, d}$, 设其信噪比为 $\gamma_{2}$, 此时采用 MRC 的接收信号可表示为:

$$
y=A y_{s, d}+B y_{r, d}
$$

其中, $A=\sqrt{P_{1} G_{s} G_{d}} h_{s, d}^{*} / N_{0}, B=\sqrt{P_{1} P_{2} G_{s} G_{d} \beta} h_{s, r}^{*} h_{r, d}^{*} / \tilde{N}_{0}$ 。假设 发送符号 $x$ 的平均功率为 1 , 则容易得到:

$$
\gamma_{1}=\frac{P_{1} G_{s} G_{d}}{N_{0}}\left|h_{s, d}\right|^{2}
$$

$$
\gamma_{2}=\frac{\frac{P_{1} G_{s} G_{r}}{N_{0}} \frac{P_{2} G_{r} G_{d}}{N_{0}}\left|h_{s, r}\right|^{2}\left|h_{r, d}\right|^{2}}{\frac{P_{1} G_{s} G_{r}}{N_{0}}\left|h_{s, r}\right|^{2}+\frac{P_{2} G_{r} G_{d}}{N_{0}}\left|h_{r, d}\right|^{2}+1}
$$

\section{3. 误符号率分析}

SER 是分析通信系统性能好坏的一个重要指标, 采 用 M-PSK 的节点在瞬时接收 SNR 情况下的条件 SER 可 表示为:

$$
P_{p s k}(\gamma) \triangleq \frac{1}{\pi} \int_{0}^{\frac{(M-1) \pi}{M}} \exp \left(-\frac{b \gamma}{\sin ^{2} \theta}\right) d \theta
$$

其中, $b=\sin ^{2}(\pi / M), \gamma$ 为信噪比。对式 (10) 取平 均值可以得到系统的平均 SER 理论值。因此, 我们容易 AF 协作方式下的闭式 SER 为: 


$$
\begin{aligned}
& P_{S E R}(\gamma)=\frac{1}{\pi} \int_{0}^{\frac{(M-1) \pi}{M}} E\left[\exp \left(-\frac{b\left(\gamma_{1}+\gamma_{2}\right)}{\sin ^{2} \theta}\right)\right] d \theta \\
& =\frac{1}{\pi} \int_{0}^{\frac{(M-1) \pi}{M}} \frac{N_{0} \sin ^{2} \theta}{N_{0} \sin ^{2} \theta+b P_{1} G_{s} G_{d} \sigma_{s, d}^{2}} \times \\
& \frac{N_{0} \sin ^{2} \theta\left(P_{1} G_{s} G_{r} \sigma_{s, r}^{2}+P_{2} G_{r} G_{d} \sigma_{r, d}^{2}\right)}{N_{0} \sin ^{2} \theta\left(P_{1} G_{s} G_{r} \sigma_{s, r}^{2}+P_{2} G_{r} G_{d} \sigma_{r, d}^{2}\right)+b P_{1} G_{s} G_{r} \sigma_{s, r}^{2} P_{2} G_{r} G_{d} \sigma_{r, d}^{2}} d \theta
\end{aligned}
$$

从式(11)我们可以看出, 系统 SER 理论值计算较为 复杂, 虽然可以通过数值计算得到结果, 但是很难分析 系统性能, 本文试图推导一个 SER 的近似表达, 从而更 好地分析基于定向天线的 AF 协作模式下的系统性能。 在 $\mathrm{AF}$ 协作模式下, 要求 $\theta>0$, 即 $0<\sin ^{2} \theta \leq 1$, 因此:

$$
\begin{aligned}
& P_{S E R}(\gamma) \leq \frac{1}{\pi} \int_{0}^{\frac{(M-1) \pi}{M}} \frac{N_{0} \sin ^{2} \theta}{b P_{1} G_{s} G_{d} \sigma_{s, d}^{2}} \\
& \times \frac{N_{0} \sin ^{2} \theta\left(P_{1} G_{s} G_{r} \sigma_{s, r}^{2}+P_{2} G_{r} G_{d} \sigma_{r, d}^{2}\right)}{b P_{1} G_{s} G_{r} \sigma_{s, r}^{2} P_{2} G_{r} G_{d} \sigma_{r, d}^{2}} d \theta
\end{aligned}
$$

通过式（12）即可以确定 SER 的一个宽松上界:

$$
\stackrel{U}{P}_{S E R}=\max \left(P_{S E R}(\gamma)\right)=C \times \frac{P_{1} G_{s} \sigma_{s, r}^{2}+P_{2} G_{d} \sigma_{r, d}^{2}}{P_{1}^{2} P_{2} G_{s}^{2} G_{r} G_{d}{ }^{2} \sigma_{s, d}^{2} \sigma_{s, r}^{2} \sigma_{r, d}^{2}}
$$

其中 $C=\frac{1}{\pi b^{2}} N_{0} \int_{0}^{2} \frac{(M-1) \pi}{M} \sin ^{4} \theta d \theta$, 可以知道, 在 $\mathrm{M}$ 给定的情 况下, $\mathrm{C}$ 为常数。

\section{4. 最优化功率分配}

本文以 SER 上界为优化目标, 引入拉格朗日乘数法 进行优化配置, 则最优化功率分配等效为:

$$
\left\{\begin{array}{c}
\arg \min \left\{P_{\text {SER }}\right\} \\
\text { s.t. } \quad P 1+P 2=P
\end{array}\right.
$$

其中, $P_{S E R}=\frac{P_{1} G_{s} \sigma_{s, r}^{2}+P_{2} G_{d} \sigma_{r, d}^{2}}{P_{1}^{2} P_{2} G_{s}^{2} G_{r} G_{d}^{2} \sigma_{s, d}^{2} \sigma_{s, r}^{2} \sigma_{r, d}^{2}}$ 。定义拉格朗日函数 为:

$$
L=P_{S E R}+\varphi(P 1+P 2-P)
$$

其中 $\varphi$ 为拉格朗日算子, 将式 (15) 分别对 $P_{1}, P_{2}$ 和 $\varphi$ 求偏导数, 并令 $\partial L / \partial P 1=0, \partial L / \partial P 2=0, \partial L / \partial \varphi=0$, 经过 整理可以得到:

$$
\left\{\begin{array}{l}
P_{1}=\frac{\sqrt{G_{d}} \sigma_{s, r}+\sqrt{G_{d} \sigma_{s, r}^{2}+8 G_{s} \sigma_{r, d}^{2}}}{3 \sqrt{G_{d}} \sigma_{s, r}+\sqrt{G_{d} \sigma_{s, r}^{2}+8 G_{s} \sigma_{r, d}^{2}}} P \\
P_{2}=\frac{2 \sqrt{G_{d}} \sigma_{s, r}}{3 \sqrt{G_{d}} \sigma_{s, r}+\sqrt{G_{d} \sigma_{s, r}^{2}+8 G_{s} \sigma_{r, d}^{2}}} P
\end{array}\right.
$$

设功率分配因子为 $\lambda$, 即 $P_{1}=\lambda P, P_{2}=(1-\lambda) P$, 则可以得到:

$$
\begin{gathered}
\lambda=\left(\sqrt{G_{d}}+\sqrt{G_{d}+8 G_{s} \frac{\sigma_{r, d}^{2}}{\sigma_{s, r}^{2}}}\right) /\left(3 \sqrt{G_{d}}+\sqrt{G_{d}+8 G_{s} \frac{\sigma_{r, d}^{2}}{\sigma_{s, r}^{2}}}\right) \\
1-\lambda=\frac{2}{3+\sqrt{1+8 G_{s} \sigma_{r, d}^{2} / G_{d} \sigma_{s, r}^{2}}}
\end{gathered}
$$

由式 (17) 可以看出, 当各结点定向增益一定时, $\sigma_{r, d}^{2} / \sigma_{s, r}^{2}$ 越大, 则 $\lambda$ 越大, 即 $P_{1}$ 更大, 因此源节点将分 得更多的发射功率。由式 (18) 可以看出, $0<1-\lambda<1 / 2$, 因此总有 $1 / 2<\lambda<1$, 当 $\sigma_{s, r}^{2} \ll \sigma_{r, d}^{2}$ 时, $1-\lambda$ 趋于零, 则 $\lambda$ 趋于 1 , 即应该将全部的功率都分给源节点, 当 $\sigma_{s, r}^{2} \gg \sigma_{r, d}^{2}$ 时, $1-\lambda$ 趋于 $1 / 2$, 则此时源与中继应该采用 等功率分配。

\section{5. 仿真分析}

本文仿真假设在 QPSK 调制下，各节点发送功率归 一化为 1 , 即 $N_{s, d}=N_{s, r}=N_{r, d}=1, \mathrm{M}=4, b=1$ 。

(1) 以定向协作 AF 闭式 SER 为研究目标考察最 优功率分配与等功率分配对系统 SER 性能影响, 令 $S N R=30 d B, G_{s}=G_{r}=G_{d}=15 d B$, 将该条件代入公式 (17) 可以得到最优功率分配系数 $\lambda=0.6667$, 从而得到图 2 。

从图 2 可以看出, 经过最优功率分配后, SER 变小, 系统性能明显变好, 最优功率分配因子虽然是基于上界 得出, 但是对于整个系统的 SER 分析仍有很强应用价 值。

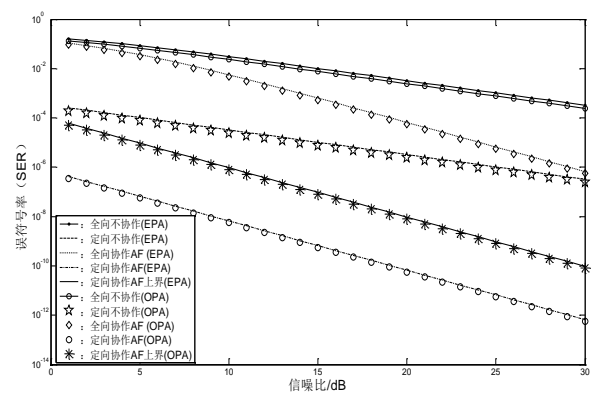

图 2 等功率分配与最优功率分配的 SER 比较 
(2)以定向协作 AF 闭式 SER 为研究目标考察功率 分配因子与定向增益对系统 SER 性能的影响。参数设 为: $h=\left[\sigma_{s, d}{ }^{2}, \sigma_{s, r}{ }^{2}, \sigma_{r, d}{ }^{2}\right]=[1,1,1], S N R=15 d B$;

(a) $G_{s}$ 变化 $=\left[G_{s}, G_{r}, G_{d}\right]=[i, 15,15](d B)$;

（b） $G_{r}$ 变化 $=\left[G_{s}, G_{r}, G_{d}\right]=[15, i, 15](d B)$;

（c） $G_{d}$ 变化 $=\left[G_{s}, G_{r}, G_{d}\right]=[15,15, i](d B)$; 其中, $0 d B \leq i \leq 15 d B$, 仿 真结果如图 3 所示。

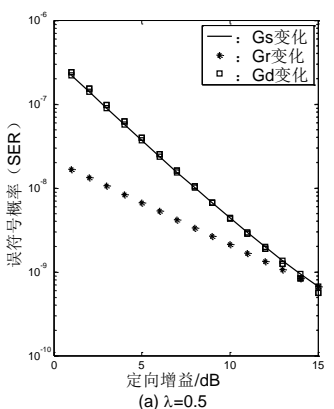

图 3 各节点定向增益与 SER 关系对比

从图 3 可知: (1)定向 $\mathrm{AF}$ 协作通信系统的误符号率随 各节点的定向增益增加而下降, 相比于中继节点定向增 益变化引起的误符号率下降, 源节点和目的节点的定向 增益变化引起的误符号率下降速度更快; (2)从图 3（a） 可以看出, 当采用等功率分配时, $G_{s}$ 和 $G_{d}$ 的 SER 变化 曲线重合在一起, 这个情况我们可以通过对式 (12) 进 行分析, 通过观察发现在等功率分配的情况下, $G_{s}$ 和 $G_{d}$ 具有对称的关系, 因此理论分析和实际仿真吻合; (3)当 采用最优功率分配则得到图 $3(\mathrm{~b}), G_{s}$ 和 $G_{d}$ 不再是对称 的关系, 二者表现为两条不同的曲线。因此, 如何在定 向增益已知的情况下, 合理分配系统总功率从而使得系 统 SER 更小就显得相当重要。

\section{6. 结束语}

本文重点研究了引入定向天线后 $\mathrm{AF}$ 协议下协作通 信系统 SER 性能。通过理论上推导引入定向天线后的 SER 表达式及其近似上界, 进一步研究最优化功率分配 问题。证明了在引入定向天线的协作通信模式下, 由于 其在特定方向上可以提供通信增益, 从而使得系统 SER 性能有较大提高; 同时, 信道质量对 SER 性能影响较大, 高效的功率分配可以显著降低资源消耗，获得更好的服
务质量, 根据信道环境进行最优功率分配比等功率分配 具有更好的 SER 性能。

\section{参考文献(References)}

[1] Ilhan, H., Akin, AI. Performance analysis of AF relaying cooperative systems with relay selection over double Rayleigh fading channels // 2012 6th International Conference on Signal Processing and Communication Systems (ICSPCS), 2012:1-6.

[2] Guo, W, Liu, J.,Zheng, L.,Xu, H.Capacity study of multiuser cooperative networks with fixed-gain relays. Networks, IET, 2012, 1(3):101-107.

[3] Shengbo Zhang, Xiang-Gen Xia, Jiangzhou Wang.Cooperative performance and diversity gain of wireless relay networks. IEEE Journal on Selected Areas in Communications, 2012, 30(9): 1623-1632.

[4] Nasir, A.A., Mehrpouyan, H., Blostein, S.D, Durrani, S. Timing and carrier synchronization with channel estimation in multi-relay cooperative networks. IEEE Transactions on Signal Processing, 2012, 60(2):793-811.

[5] Min Zhou, Qimei Cui, Jantti, R,Xiaofeng Tao. Energy-efficient relay selection and power allocation for two-way relay channel with analog network coding. IEEE, Communications Letters, 2012, 16(6):816-819.

[6] Li Li, Texas, Denton, Shengli Fu. Cooperative communication based on random beamforming strategy in wireless sensor networks //Global Communications Conference (GLOBECOM), 2012:4108-4113.

[7] Yong Li,Zhaocheng Wang,Depeng Jin,Lieguang Zeng. Collaborative vehicular content dissemination with directional antennas. IEEE Transactions on Wireless Communications, 2012 , 11(4): 1301-1306.

[8] Ling Ding, Weili Wu, Willson, J, Hongjie Du. Efficient virtual backbone construction with routing cost constraint in wireless networks using directional antennas. IEEE Transactions on Mobile Computing, 2012, 11(7): 1102 - 1112.

[9] Abdullah, A. A, Lin Cai, Gebali, F. DSDMAC: dual sensing directional MAC protocol for Ad Hoc networks with directional antennas. IEEE Transactions on Vehicular Technology, 2012, 61(3): 1266-1275.

[10] Wang Xu-hua, Bai Peng, Li Ming-yang, Li Huan-yu, Lin Jin-fu. Performance analysis of multi-relays AF cooperative communication system based on directional antenna. Systems Engineering and Electronic, 2013, 35(3): 624-628. 